\section{Behandlung der Herzinsuffizienz}

\author{
J. Bauersachs ${ }^{1}$ \\ ${ }^{1}$ Direktor der Klinik für Kardiologie und Angiologie, \\ Medizinische Hochschule Hannover
}

Die Herzinsuffizienz ist trotz großer therapeutischer Fortschritte weiterhin eine Erkrankung mit hoher Prävalenz und Inzidenz. Die Herzinsuffizienz ist der häufigste Grund für Krankenhauseinweisungen; jährlich werden in Deutschland ca. 400000 Patienten wegen Herzinsuffizienz im Krankenhaus behandelt, 50000 versterben daran.

Bis vor wenigen Jahrzehnten bestand die Therapie der Herzinsuffizienz weitgehend aus Diuretika und Digitalis. Die präklinische und klinische Forschung hat jedoch in den letzten 50 Jahren zur Einführung von mehreren Medikamentenklassen geführt, welche die Morbidität und Mortalität bei der systolischen Herzinsuffizienz drastisch verbessert haben. Darüber hinaus ist die Device-Therapie zu nennen, insbesondere implantierbare Defibrillatoren sowie die Resynchronisationstherapie (CRT), durch welche eine weitere entscheidende Verbesserung der Symptomatik und Prognose erzielt werden konnte.

Die Optimierung der medikamentösen Therapie der systolischen Herzinsuffizienz in den letzten Jahrzehnten beruhte vor allem auf einem besseren pathophysiologischen Verständnis der Krankheit. Vom ursprünglich kardiorenalen Modell der Herzinsuffizienz kam man in den Sechziger- und Siebzigerjahren zum hämodynamischen Modell; die Entwicklung positiv inotroper Substanzen war jedoch nicht mit einer signifikanten Verbesserung des Überlebens verbunden. Erst durch das pathophysiologische Konzept des linksventrikulären Remodelings und der neurohormonalen Aktivierung konnte auf der Basis von in Tiermodellen entwickelten therapeutischen Ansätzen in zahlreichen klinischen Studien eine medikamentöse Kombinationstherapie der systolischen Herzinsuffizienz erarbeitet werden, die heute der Evidenz-basierte Standard ist.

Zunächst konnte mit Hemmstoffen des Angiotensin Converting-Enzyms (ACE) gezeigt werden, dass die Erweiterung der linken Herzkammer nach einem großen Herzinfarkt deutlich verringert wurde. In translationalen klinischen Studien wurde mit ACE-Hemmern bei Patienten mit systolischer Herzinsuffizienz eine hochsignifikante Reduktion der Mortalität und der Krankenhausaufnahmen wegen Herzinsuffizienz erreicht. Die zusätzliche Gabe von MineralocorticoidrezeptorAntagonisten (Spironolacton, Eplerenon) führte zu einer additiven Verbesserung sowohl des linksventrikulären Remodelings in Tiermodellen als auch des Überlebens und der Rate an Krankenhauseinweisungen bei Patienten mit systolischer Herzinsuffizienz.

Während die Betablocker über Jahrzehnte bei der systolischen Herzinsuffizienz aufgrund ihrer negativ-inotropen Wirkung als kontraindiziert galten, konnte durch mehrere klinische Studien in den Neunzigerjahren nachgewiesen werden, dass eine Therapie mit Betablockern (nach Beginn mit niedriger Dosis und Dosissteigerung über Wochen bis Mona- te) zu einer hochsignifikanten Verminderung der Mortalität und auch zu einer Verbesserung der Herzerweiterung und der linksventrikulären Auswurffraktion führt. Auf dieser Basis konnte auch für den Sinusknotenhemmer Ivabradin gezeigt werden, dass eine Verringerung der Herzfrequenz zu einer Prognoseverbesserung führt.

Außerdem ist kürzlich mit Valsartan/Sacubitril der erste Vertreter einer neuen Klasse der Angiotensin-Rezeptor/Neprilysin-Inhibitoren in die Therapie der systolischen Herzinsuffizienz eingeführt worden. Diese Substanz wird vermutlich mittelfristig den ACE-Hemmer in der Therapie der Herzinsuffizienz ersetzen, nachdem in der PARADIGM-Studie nachgewiesen werden konnte, dass im Vergleich zur Standardbehandlung mit dem ACE-Hemmer Enalapril die Behandlung mit Sacubitril/Valsartan zu einer weiteren Verminderung von Mortalität und Krankenhausaufnahmen bei systolischer Herzinsuffizienz führt.

Auch wenn durch die ausgefeilte medikamentöse Kombinationstherapie die Prognose der systolischen Herzinsuffizienz in den letzten Jahrzehnten dramatisch verbessert werden konnte, gibt es jedoch immer noch zahlreiche Patienten mit fortgeschrittener Herzinsuffizienz, die aufgrund von Hypotonie, Niereninsuffizienz, Hyperkaliämie nicht mit den Zieldosierungen der ACE-Hemmer, Betablocker und Mineralocorticoidrezeptor-Antagonisten behandelt werden können. Bei diesen Patienten könnte die Therapie mit Digitalis immer noch eine hilfreiche Rolle spielen. In der BMBF-geförderten DIGIT HF-Studie untersuchen wir aktuell, ob die Gabe von Digitoxin bei fortgeschrittener Herzinsuffizienz mit einer Verbesserung der Prognose verbunden ist.

Trotz der erheblichen Fortschritte in der Behandlung der Herzinsuffizienz ist die Inzidenz und Prävalenz der Herzinsuffizienz weiterhin hoch, zum einen aufgrund der demografischen Entwicklung mit älterwerdender Bevölkerung, zum anderen aufgrund von durch optimierte Intensivmedizin verbessertem Überleben auch bei schwerer akuter Form der Herzinsuffizienz wie dem akuten Myokardinfarkt. Daher sind weitere Forschungsanstrengungen nötig, um den Patienten mit Herzinsuffizienz noch besser helfen zu können. Dies gilt insbesondere auch für die zahlreichen Patienten mit diastolischer Herzinsuffizienz bei erhaltener linksventrikulärer Ejektionsfraktion, für die es leider nahezu keine prognoseverbessernden Medikamente gibt.

Persönlich habe ich mit meiner Forschung im Bereich der Herzinsuffizienz im hohen Maße von der Förderung durch die Deutsche Forschungsgemeinschaft seit 20 Jahren profitiert, insbesondere in den Sonderforschungsbereichen 355 und 688 in Würzburg, im Exzellenzcluster REBIRTH in Hannover und jetzt auch in unserer gerade bewilligten Klinischen Forschergruppe KFO 311 „(Prä)-terminale Herz- und Lungeninsuffizienz: Entlastung und Reparatur“ an der Medizinischen Hochschule Hannover. Interessante therapeutische Ansätze, die möglicherweise in neue Herzinsuffizienz-Medikamente münden könnten, sind beispielsweise die nicht-codierenden RNAs, zum einen microRNAs, zum anderen sogenannte long non-coding RNAs.

Interessenkonflikte: Forschungsunterstützung durch das Bundesministerium für Bildung und Forschung für die DIGIT HF-Studie, außerdem von den Firmen Bayer, Abiomed, Med- 
tronic, Biotronik. Vortragshonorare bzw. Beratertätigkeit von den Firmen Novartis, Pfizer, Bayer, Servier, Orion, St. Jude Medical, Thoratec, HeartWare

Bibliografie

DOI http://dx.doi.org/10.1055/s-0042-114462

Drug Res 2016; 66, Suppl. 1: S24-S25

(c) Georg Thieme Verlag KG Stuttgart · New York .

ISSN 2194-9379
Korrespondenzadresse

Prof. Dr. med. Johann Bauersachs

Direktor der Klinik für Kardiologie

und Angiologie

Medizinische Hochschule Hannover

Carl-Neuberg-Straße 1

30625 Hannover

bauersachs.johann@mh-hannover.de

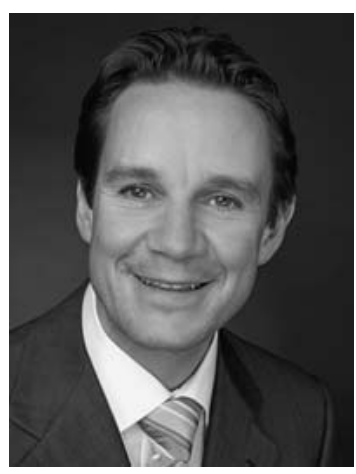

